\title{
A proper integral for the electric field at the surface of a conducting sphere
}

\author{
F. M. S. Lima*1@ \\ ${ }^{1}$ Universidade de Brasília, Instituto de Física, 70919-970, Brasília, DF, Brasil.
}

Received on May 6, 2020. Revised on July 11, 2020. Accepted on July 27, 2020.

\begin{abstract}
In a recent paper, I have shown that the electric field at the surface of a charged conducting sphere evaluates to half the field discontinuity across the surface of the sphere. This exact result has been found by solving an improper integral, which has been criticized by Assad in a very recent paper in this journal [6]. In this note, I present a simpler approach that yields a proper integral for that field. This integral also evaluates to exactly half the field discontinuity, contrarily to Assad's objections.
\end{abstract}

Keywords: Electrostatic fields, Gauss's law, Conducting sphere.

On seeking for a deeper understanding of a scientific topic, we often formulate simple questions which, though not realizable experimentally, should be satisfactorily answered. The electric field at the surface of a charged conducting sphere in electrostatic equilibrium, mentioned in virtually all introductory physics and electromagnetism textbooks, is among such topics because it is impossible to make a perfectly-shaped sphere ${ }^{1}$ Despite this practical impossibility, the field at the surface of an ideal, perfect sphere with radius $R$ and charge $Q$ may, a priori, be determined mathematically. As the net charge on any bulk conductor must reside on its surface, it follows from Gauss's law that the magnitude of the field of a conducting sphere must leap from a null value inside the sphere to $\sigma / \epsilon_{0}=k Q / R^{2}$, just outside it (see, e.g., Refs. 2 2 4 $)^{2}$ Here, $\sigma=Q /\left(4 \pi R^{2}\right)$ is the areal charge density on the surface of the sphere and $k=1 /\left(4 \pi \epsilon_{0}\right)$ is the Coulomb constant, $\epsilon_{0}$ being the electric permittivity of free space. For points located exactly at the surface, however, the amount of charge surrounded by a spherical gaussian surface with radius $R$ is ambiguous, so Gauss's law is inconclusive. In a previous work, by treating the charge distribution as a collection of thin charged rings I have already shown that the field at the surface evaluates to $\frac{1}{2} k Q / R^{2}=\sigma /\left(2 \epsilon_{0}\right)$, i.e. half the field discontinuity across the surface [5]. As this result has been found by solving an improper integral, it has been criticized in a very recent paper [6]. I present below a simpler approach that yields a proper integral for that field.

\footnotetext{
*Correspondence email address: fabio@fis.unb.br

${ }^{1}$ Even the surface of a polished metal, which seems smooth for the naked-eye, reveals a number of roughnesses under a microscope, with its surface being at an average distance $R$ to the center 1 . 2 According to Gauss's law, the electric field of a uniformly-charged spherical shell is the same as that of a conducting sphere, both inside and outside it.
}

Theorem. For a conducting sphere with radius $R$ and net charge $Q$, in electrostatic equilibrium, the electric field in any point $P$ on its surface is given by

$$
\mathbf{E}=\frac{1}{2} \frac{k Q}{R^{2}} \hat{\mathbf{r}}=\frac{\sigma}{2 \epsilon_{0}} \hat{\mathbf{r}}
$$

where $\sigma=Q /\left(4 \pi R^{2}\right)$ and $\hat{\mathbf{r}}$ is the radial (outward) unit-vector at point $P$.

Proof. Without loss of generality, take $Q>0$ and the $z$-axis along the line from the center, at $x=y=z=0$, to point $\mathrm{P}$, at $x=y=0$ and $z=R$, as seen in Fig. 1 . below. On treating the spherical charge distribution as a collection of horizontal circular rings with radius $r$, varying from 0 to $R$, and variable charge $d Q=\sigma d A=$ $\sigma(2 \pi r d s)$, where $d s=R d \theta$ is the width of each ring, $\theta$ being the polar angle (in spherical coordinates), we begin deriving the electric field $d \mathbf{E}$ created in $\mathrm{P}$ by the charged ring centered at point $(0,0, z)$, with $-R \leq$ $z<+R$, which lies at a distance $R-z$ from $\mathrm{P}$, as indicated in Fig. 1. The azimuthal symmetry of the charge distribution guarantees that the field in $\mathrm{P}$ must point along the $z$-axis direction, so $d \mathbf{E}=d E_{z} \hat{\mathbf{k}}$, where $\hat{\mathbf{k}}$ is the unit-vector for the $z$-axis and

$$
d E_{z}=k \frac{(R-z)}{\left[r^{2}+(R-z)^{2}\right]^{\frac{3}{2}}} d Q,
$$

according to the well-known formula for the field of a uniformly-charged ring, as given e.g. in Eq. (22-16) of Ref. [4]. Note that this expression is valid for all $-R \leq$ $z<+R$, being an indeterminate form of the kind ' $0 / 0$ ' for $z=R$. A direct integration $E_{z}=\int d E_{z}$ would lead us to an improper integral, as done in Ref. [5], so, in view to change it into an equivalent proper integral, let us express $d E_{z}$ as a function of $\theta$ only (apart from multiplicative 


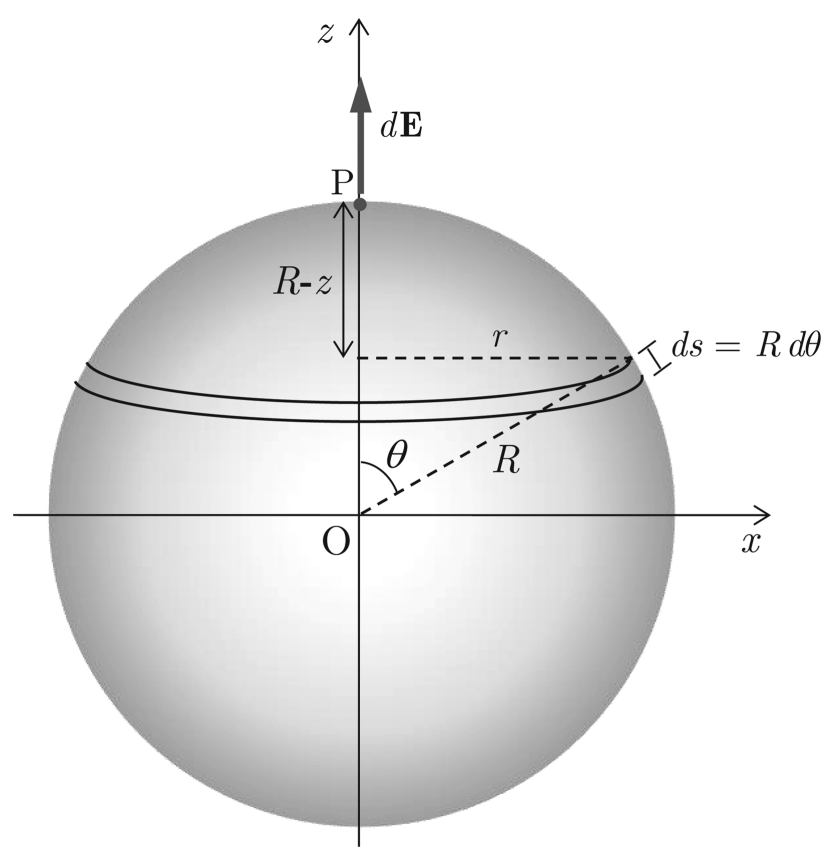

Figure 1: A conducting sphere with radius $R$ and electric charge $Q>0$ is cut into a large number of thin circular rings of radius $r=R \sin \theta$, each having a charge $d Q=\sigma(2 \pi r d s)$. Each ring produces a vertical electric field $d \mathbf{E}=d E_{z} \hat{\mathbf{k}}$ at point $\mathrm{P}$.

constants). Since $z=R \cos \theta$ and $r=R \sin \theta$, one finds

$$
\begin{aligned}
d E_{z} & =2 \pi k \sigma \frac{R^{2} \sin \theta R(1-\cos \theta)}{\left[R^{2} \sin ^{2} \theta+R^{2}(1-\cos \theta)^{2}\right]^{\frac{3}{2}}} d \theta \\
& =2 \pi k \sigma \frac{\sin \theta(1-\cos \theta)}{\left[\sin ^{2} \theta+(1-\cos \theta)^{2}\right]^{\frac{3}{2}}} d \theta \\
& =2 \pi k \sigma \frac{\sin \theta(1-\cos \theta)}{(2-2 \cos \theta)^{\frac{3}{2}}} d \theta \\
& =\frac{\pi}{\sqrt{2}} k \sigma \frac{\sin \theta}{\sqrt{1-\cos \theta}} d \theta
\end{aligned}
$$

Though this last expression is still an indeterminate form of the kind ' $0 / 0$ ' for $\theta=0$ (which corresponds to $z=R) 3^{3}$ the trigonometric identities $\sin \theta=$ $2 \sin (\theta / 2) \cos (\theta / 2)$ and $\sin ^{2}(\theta / 2)=(1-\cos \theta) / 2$ allow us to simplify it to

$$
\begin{aligned}
d E_{z} & =\pi k \sigma \frac{\sin (\theta / 2) \cos (\theta / 2)}{\sqrt{\sin ^{2}(\theta / 2)}} d \theta \\
& =\pi k \sigma \cos \left(\frac{\theta}{2}\right) d \theta
\end{aligned}
$$

which is a well-defined function for $\theta=0$, being bounded and continuous there at that point. The integration over

\footnotetext{
${ }^{3}$ Note that $\lim _{\theta \rightarrow 0} \frac{\sin \theta}{\sqrt{1-\cos \theta}}=\sqrt{2}$, rather than $\infty$, as would be the case for the Coulombian field from a finite point charge $Q$.
}

all rings then yields

$$
E_{z}=\pi k \sigma \int_{0}^{\pi} \cos \left(\frac{\theta}{2}\right) d \theta
$$

which is a proper integral, since the integrand remains bounded and continuous throughout the integration interval, including its endpoints. On substituting $u=\theta / 2$, one promptly finds

$$
\begin{aligned}
E_{z} & =2 \pi k \sigma \int_{0}^{\pi / 2} \cos u d u \\
& =\frac{\sigma}{2 \epsilon_{0}} .
\end{aligned}
$$

We emphasize that the function at the right-hand side of Eq. (1) is undefined only at the point $z=R$, where it returns the indeterminate form ' $0 / 0$ ', remaining bounded and continuous for all other points in the domain $-R \leq z \leq+R$. Then, we have excluded that point from the domain, but we have properly included it there in Eq. (3), which has led to an elementary, proper integral in Eq. (4). Mathematically, this can be viewed as a regularization of the improper Riemann integral solved in Ref. [5], which is a valid procedure since the value of the integral of a bounded function that is continuous in an interval of integration, except at a point belonging to this interval, does not change when we remove this point from the integration domain, as follows from a well-known theorem by Cauchy (see, e.g., Theorem 8.1 and Sec. 8.4, as well as Ex. 8.4.6, of Ref. 7 ) ${ }_{4}^{4}$ Physically speaking, this regularization is justified by noting that the charge is distributed uniformly on the surface of the sphere, with a constant density $\sigma$, so the charge $d Q=\sigma d A=2 \pi \sigma R^{2} \sin \theta d \theta$ in each ring will tend to zero as $r \rightarrow 0$ (i.e., when $z \rightarrow R^{-}$or $\theta \rightarrow 0$ ), which means that our point $\mathrm{P}$, which encloses a null area, does not enclose a finite amount of charge (i.e., it cannot be treated as a finite point charge). Therefore, the expression for $d E_{z}$ in Eq. (1) does not present a non-integrable singularity at $z=R$ (i.e., at $\theta=0$ ), which shows that Assad's argument below Eq. (15) of Ref. [6] is incorrect. In fact, even an older argument by Assad, as found at the end of Ref. [9], that the electric field is undefined at the surface of a conducting sphere because there is electric charge there, is also incorrect. The presence of an electric charge $d Q$ at a point does not always create an infinite electric field there, otherwise the well-known result for the field inside a uniformly-charged sphere, namely $E(r)=\left(k Q / R^{3}\right) r$, valid for $0 \leq r \leq R$, as found, e.g., in Eq. (23-20) of Ref. [4], or Ex. 23.6(B) of Ref. [10], would not make sense!

The electric field is then discontinuous at the surface of a conducting sphere, leaping from 0 to $\sigma /\left(2 \epsilon_{0}\right)$

\footnotetext{
${ }^{4}$ For a simple but rigorous proof of this property of Riemann integrals, see Ref. 8.
} 
when we pass from points inside the sphere to any point at its surface, and then from this to $\sigma / \epsilon_{0}$ for points just outside the sphere. Interestingly, the half-factor in our theorem also arises in more elaborate microscopic (quantum-mechanical) models, in a scale in which both the charge distribution and the electric field change continuously from inside to just outside a charged conductor [11]. According to these models, a thin transition slab with extension of a few atomic diameters is formed within which the field increases smoothly from nearly zero, inside the conductor, to its maximum value $\sigma / \epsilon_{0}$, attained at a point about $4 \AA$ outside the conductor, as shown in Fig. I.5 of Ref. 11. There in that figure, the field at the surface $(x=0)$ clearly equals $0.5 \sigma / \epsilon_{0}$.

In summary, though Gauss's law promptly reveals a leap discontinuity from 0 to $\sigma / \epsilon_{0}$ in the electric field when we cross the surface of a charged conducting sphere, it does not provide a definite result for the field at this surface. In order to fill this gap, in this note I have shown, within the rigour of calculus, that this field evaluates to half that discontinuity. Our proof involves only basic integration rules, so it could well be adopted in introductory physics classes and textbooks.

\section{References}

[1] D.J. Whitehouse, Handbook of Surface and Nanometrology (CRC Press, Boca Raton, 2011), 2nd ed.

[2] H.D. Young and R.A. Freedman, University Physics (Pearson, New York, 2016), 14th ed.

[3] D.J. Griffiths, Introduction to Electrodynamics (Pearson, New York, 2013), 4th ed.

[4] D. Halliday, R. Resnick and J. Walker, Fundamentals of Physics (Wiley, New York, 2014), 10th ed.

[5] F.M.S. Lima, Resonance 23, 1215 (2018).

[6] G.E. Assad, Rev. Bras. Ens. Fís. 42, e20190245 (2020).

[7] B.S. Thomson, J.B. Bruckner and A.M. Bruckner, Elementary Real Analysis (Prentice Hall, Upper Saddle River, 2008), 2nd ed.

[8] Changing one point does not change the Riemann integral, available in: https://math.stackexchange. com/questions/857961/changing-one-point-doesnot-change-the-riemann.

[9] G.E. Assad, Rev. Bras. Ens. Fís. 34, 4701 (2012).

[10] R.A. Serway and J.W. Jewett Jr., Physics for Scientists and Engineers (Cengage, Boston, 2019), 10th ed.

[11] J.D. Jackson, Classical Electrodynamics (Wiley, New York, 1999), 3rd ed. 